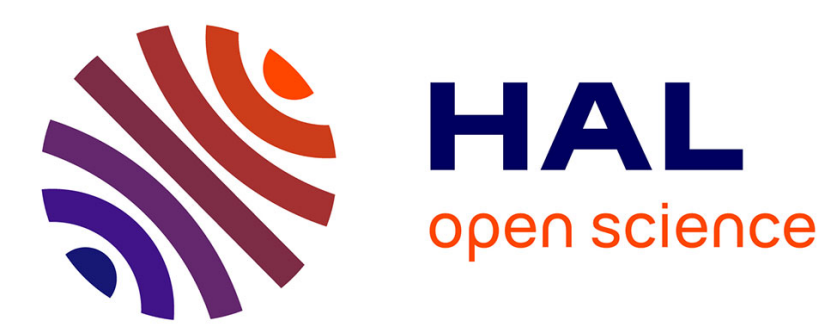

\title{
Computation of Solid-State Vibrational Circular Dichroism in the Periodic Gauge
}

\author{
Sascha Jähnigen, Anne Zehnacker, Rodolphe Vuilleumier
}

\section{To cite this version:}

Sascha Jähnigen, Anne Zehnacker, Rodolphe Vuilleumier. Computation of Solid-State Vibrational Circular Dichroism in the Periodic Gauge. Journal of Physical Chemistry Letters, 2021, pp.72137220. 10.1021/acs.jpclett.1c01682 . hal-03311633

\section{HAL Id: hal-03311633 \\ https://hal.science/hal-03311633}

Submitted on 1 Aug 2021

HAL is a multi-disciplinary open access archive for the deposit and dissemination of scientific research documents, whether they are published or not. The documents may come from teaching and research institutions in France or abroad, or from public or private research centers.
L'archive ouverte pluridisciplinaire HAL, est destinée au dépôt et à la diffusion de documents scientifiques de niveau recherche, publiés ou non, émanant des établissements d'enseignement et de recherche français ou étrangers, des laboratoires publics ou privés. 


\title{
Computation of Solid-State Vibrational
}

\section{Circular Dichroism in the Periodic Gauge}

\author{
Sascha Jähnigen, ${ }^{*, \dagger, \ddagger}$ Anne Zehnacker, ${ }^{\dagger}$ and Rodolphe Vuilleumier,,‡ \\ †Institut des Sciences Moléculaires d'Orsay (ISMO), CNRS, Université Paris-Saclay, \\ 91405 Orsay, France \\ $\ddagger$ PASTEUR, Département de Chimie, Ecole Normale Supérieure, PSL University, \\ Sorbonne Université, CNRS, 75005 Paris, France \\ E-mail: sascha.jahnigen@ens.psl.eu; rodolphe.vuilleumier@ens.psl.eu
}




\begin{abstract}
We introduce a new theoretical formalism to compute solid-state vibrational circular dichroism (VCD) spectra from molecular dynamics simulations. Having solved the origin-dependence problem of the periodic magnetic gauge, we present IR and VCD spectra of $(1 S, 2 S)$-trans-1,2-cyclohexanediol obtained from first-principles molecular dynamics calculations and nuclear velocity perturbation theory, along with the experimental results. As the structure model imposes periodic boundary conditions, the common origin of the rotational strength has hitherto been ill-defined and was approximated by means of averaging multiple origins. The new formalism relies on the velocity representation of VCD and the time-correlation function, whose symmetry properties are exploited to reconnect the periodic model with the finite physical system. It restores the gauge freedom of finite models, but still allows fully accounting for non-local spatial coupling terms from the gauge transport term. We show that even for small simulation cells the rich nature of solid-state VCD spectra found in experiments can be reproduced to a very satisfactory level. While the general workflow to compute solid-state VCD spectra relies on the interplay of experimental data and theoretical simulation, expressions of VCD in liquid state and for isolated systems can be derived as simplification of the general equations.
\end{abstract}

\title{
Graphical TOC Entry
}

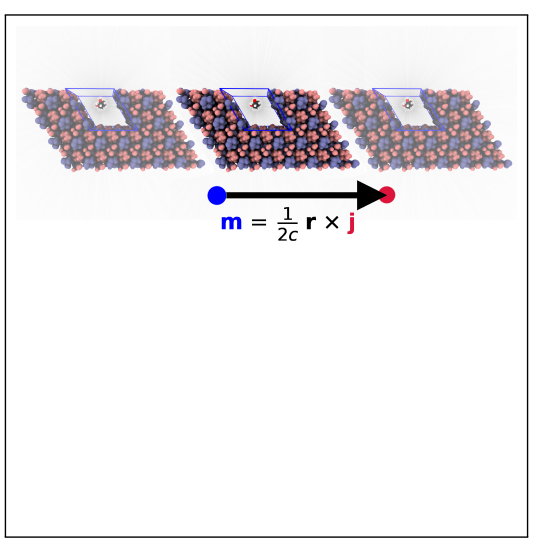




\section{Keywords}

solid-state VCD, first-principles molecular dynamics, nuclear velocity perturbation theory, cyclohexanediol 
Vibrational circular dichroism (VCD) is an increasingly popular spectroscopic technique that denotes chiral infrared (IR) spectroscopy, defined as the differential absorption of leftand right-circularly polarised light. ${ }^{1-4}$ In contrast to electronic circular dichroism, VCD is bound to electronic and nuclear degrees of freedom alike. Yet being a ground state property, it does not require the presence of separate UV-visible chromophore. Nowadays, modern spectrometers allow for an easy recording of VCD even though its intensity usually amounts to only $10^{-6}$ to $10^{-4}$ of that of IR absorption; VCD has become an invaluable tool for the non-invasive determination of absolute configurations. ${ }^{1,2,5,6}$ Its applicability, however, reaches beyond as it includes the analysis of natural products, ${ }^{7-9}$ encapsulation processes,${ }^{10-13}$ nanoparticles or catalysts, ${ }^{14-17}$ liquid crystals, ${ }^{18,19}$ and structural aspects of proteins. ${ }^{20-22}$ The outstanding conformational sensitivity is boon and bane of the VCD experiment: While a molecular substance can be characterised by VCD in terms of its conformations and non-covalent interactions, subtle conformational changes have the capacity to invert the sign of peaks, which is further complicated for superposed signals. ${ }^{23-25}$ Hence, accurate computations are indispensable for the interpretation of VCD. ${ }^{1}$ These have to properly sample the energy landscape of the system to not only get absorption frequencies right, but more importantly to correctly describe the conformational dispersion within intra- and intermolecular space. ${ }^{23}$ We recently published a survey on different computational tools to describe conformational sampling. ${ }^{24}$ Typically, VCD is measured in liquid state or in or in isolated conditions such as noble gas matrices, ${ }^{26-30}$ which is a largely standardised procedure, but requires sample solubility and may exhibit spurious solvent effects. However, in analogy to IR absorption, VCD can also be measured conveniently in solid state. ${ }^{31-35}$ This circumvents the necessity of substrate solubility since the measurements are carried out directly on (powdered) microcrystals dispersed in a matrix material, typically KBr. Spurious effects from the matrix are rare, but one has to deal with scattering effects and remnants of linear dichroism, ${ }^{2,36}$ for the elimination of which the experimental procedures have been devised, though. ${ }^{37,38}$ Deprived of conformational flexibility in the packed solid, molecules ex- 
hibit sharper and more intense VCD signals than in solution. ${ }^{2}$ At the same time, VCD can serve as a probe of crystal polymorphism as it contains information beyond local molecular signatures adding important non-local contributions from packing effects. ${ }^{11,39-41}$ We have recently shown that strongly delocalised modes lead to VCD enhancement in crystals of L-alanine determined by the chiral space group $P 2_{1} 2_{1} 2_{1} \cdot{ }^{31}$

Dealing with solid-state VCD today mainly challenges the theoretical treatment given the large system size and the ubiquitous presence of non-covalent interactions. Conventional simulations of VCD account for individual molecules, isolated or incorporated in a (model) solvent, but it is inconceivable to theoretically study an entire microcrystal. In cases where VCD is determined by non-covalent interactions with close neighbours only, isolated subdomains extracted from the crystal bulk have successfully been used in cluster or fragment studies. ${ }^{11,41-43}$ Yet, general applicability is offered by structural models that adhere to the crystallographic information and make use of periodic boundary conditions (PBC). In this letter, we present the adapted computational protocol to compute VCD in solid state within periodic boundaries. Briefly outlining the problem of defining a magnetic gauge within an infinite lattice model, we offer a solution that restores the original gauge freedom of VCD of the finite world. As proof of principle, we measured and computed the solid-state IR and VCD spectrum of (1S,2S)-trans-1,2-cyclohexanediol, denoted here as (1S,2S)-CHDO, using first principles molecular dynamics (FPMD) simulations and nuclear velocity perturbation theory (NVPT), following the general workflow presented in Scheme 1.

The theoretical protocol to a (Hessian-free) calculation of VCD comprises three steps: molecular dynamics (MD), electromagnetic response, and time-correlation within a common magnetic gauge. ${ }^{44-46}$ With MD a statistical ensemble of the system is generated containing atom positions and velocities, while response calculations yield the electromagnetic dipole moments on the time domain. Eventually, VCD is computed based on the Fourier transform of the time-correlation function (TCF) that duly accounts for the magnetic gauge. ${ }^{47}$ If an infinite lattice model is to be used, periodicity has to be reflected in the nuclear and electronic 


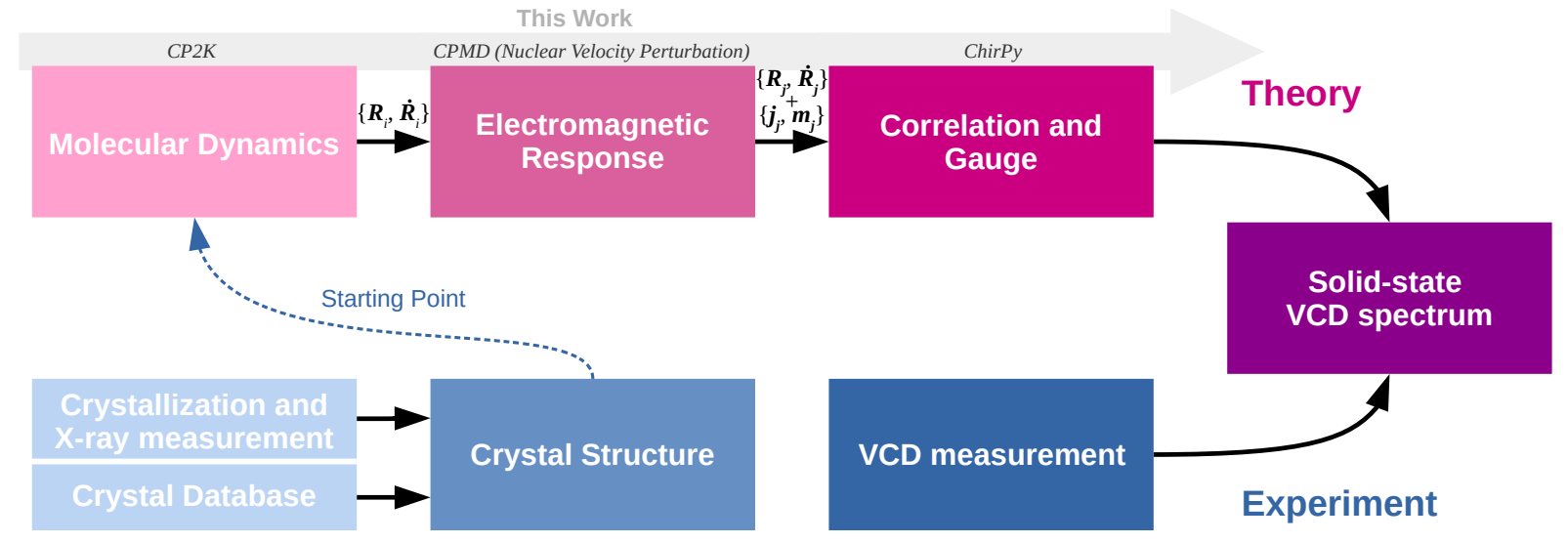

Scheme 1: Theoretical (magenta) and experimental (blue) workflow to obtain a solid-state VCD spectrum. An important ingredient is the definition of the starting point for the MD simulation, based on the experimental crystal structure. This letter focuses on "Correlation and Gauge" as it contains a fundamental problem that is encountered under periodic boundary conditions: defining a common origin for the magnetic gauge. The grey arrow icon indicates the programme packages that have been used in this work.

degrees of freedom, and this in all three steps of the theoretical protocol. An important supplement in Scheme 1 with respect to conventional calculations is the definition of the starting point of the MD simulation based on the experimental crystal structure taken from a database or crystallographic experiments. When several polymorphs exist, it is of prime importance to select the one that corresponds to the experiment; the crystal phase does usually not transform and ergodicity is readily reached for a single modification. Hence, due to the limited conformational freedom of the solid state, convergence of the calculations can be reached faster, which is a major advantage when FPMD calculations limited to rather short trajectories are employed.

The electromagnetic response is obtained classically for the nuclei, but quantum mechanically for the electrons. ${ }^{2,47}$ Yet, a standard quantum or DFT calculation relies on the Born-Oppenheimer (BO) approximation and therefore has a vanishing electronic contribution to the magnetic dipole moment. ${ }^{48}$ In order to reach "beyond-BO" and to calculate VCD, there exist two complementary formulations of quantum linear response theory: magnetic field perturbation (MFPT) ${ }^{49-51}$ yielding the magnetic susceptibility of the electronic wave 
function, and nuclear velocity perturbation (NVPT), ${ }^{52-54}$ delivering the electronic current density from which the induced magnetic moment can be obtained. Kirchner and co-workers introduced a semi-classical approach that aims at reconstructing the magnetic response from the time-dependent electron density. ${ }^{55}$ While any scheme for steps 1 or 2 can be used before going over to step 3, we rely on NVPT here as it is adapted to accurately sampling the phase space of the FPMD trajectory. ${ }^{47,53}$

"Correlation and Gauge" in Scheme 1 is the focus of this letter as it connects to a fundamental problem that is encountered under periodic boundary conditions: defining a common origin for the magnetic gauge. According to the Rosenfeld equation, VCD is proportional to the rotational strength, $R_{i f}=\operatorname{Im}[\langle i|\hat{\boldsymbol{\mu}}| f\rangle \cdot\langle f|\hat{\mathbf{m}}| i\rangle] .{ }^{56}$ It depends on the vibronic transition $|i\rangle \rightarrow|f\rangle$, where $\hat{\boldsymbol{\mu}}$ and $\hat{\mathbf{m}}$ denote the electric and magnetic dipole moment operator, respectively. $R_{\text {if }}$ can alternatively be expressed using the current dipole moment, $\hat{\mathbf{j}}=\hat{\dot{\boldsymbol{\mu}}}=d \hat{\boldsymbol{\mu}} / d t$, through the hypervirial theorem, $\langle i|\hat{\mathbf{j}}| f\rangle=\mathrm{i} \omega_{i f}\langle i|\hat{\boldsymbol{\mu}}| f\rangle .{ }^{57}$ Thus assuming its "velocity form", the differential molar absorption coefficient for VCD reads in the Schrödinger picture: ${ }^{2}$

$$
\Delta \varepsilon(\omega)=\frac{16 \pi^{2}}{3 N \hbar c n(\omega)}\left(1-e^{-\beta \hbar \omega}\right) \sum_{f i} \rho_{i} \operatorname{Re}[\langle i|\hat{\mathbf{j}}| f\rangle \cdot\langle f|\hat{\mathbf{m}}| i\rangle] \delta\left(\omega_{f i}-\omega\right)
$$

(using Gaussian-cgs units) at temperature $T=1 / k_{B} \beta$ with the density of initial states $\rho_{i}$, the number of units $N$ and the refraction index of the medium $n$. Note the division factor of 3 from isotropically averaging over all spatial orientations. ${ }^{36}$ When used in combination with molecular dynamics simulations, $\Delta \varepsilon(\omega)$ can be expressed in the Heisenberg picture in terms of the quantum TCF, $C(t)=\langle\hat{\mathbf{j}}(0) \hat{\mathbf{m}}(t)\rangle:^{44}$

$$
\Delta \varepsilon(\omega)=\frac{16 \pi^{2}}{3 N \hbar c n(\omega)}\left(1-e^{-\beta \hbar \omega}\right) \operatorname{Re}\left[\frac{1}{2 \pi} \int_{-\infty}^{\infty} d t e^{-i \omega t}\langle\hat{\mathbf{j}}(0) \hat{\mathbf{m}}(t)\rangle\right]
$$

The two formulations of VCD are in full analogy to vibrational absorption. ${ }^{46,47,58}$ Likewise, the actual advantage of using Equation 2 lies in taking the classical limit of the quantumcorrelation function, $C(t) \rightarrow C_{\mathrm{cl}}(t)=\langle\mathbf{j}(0) \mathbf{m}(t)\rangle$, that is, to employ the computationally 
affordable classical time-correlation function $C_{\mathrm{cl}}$ instead. ${ }^{45}$ Conveniently, the quantum correction for the classical treatment of the nuclei can be added a posteriori and is carried out based on the classical limit of the Kubo-transformed time-correlation function. ${ }^{45-47,59}$ With this, the expression of the differential molar absorption coefficient becomes

$$
\Delta \varepsilon(\omega) \approx \frac{8 \pi \beta \omega}{3 N c n(\omega)} \int_{-\infty}^{\infty} d t e^{-i \omega t}\langle\mathbf{j}(0) \mathbf{m}(t)\rangle
$$

It is important to note that also in solid-state calculations isotropic conditions are generated (see the SI for details of the experiment), which eliminates any contribution from electric quadrupole moments. ${ }^{36}$

The magnetic dipole moment is defined with respect to an origin, which defines a gauge. Being an axial vector it transforms upon spatial translation from one origin $\mathcal{O}$ to another $\mathcal{O}^{\prime}$, leading to the well-know equality,

$$
\mathbf{m}^{\mathcal{O}^{\prime}}=\mathbf{m}^{\mathcal{O}}+\frac{1}{2 c}\left(\mathcal{O}-\mathcal{O}^{\prime}\right) \times \mathbf{j}(t)
$$

In finite systems this gauge dependence naturally vanishes in the velocity form of the rotational strength, ${ }^{2}$ likewise for $C_{\mathrm{cl}}$ :

$$
C_{\mathrm{cl}}^{\mathcal{O}^{\prime}}=C_{\mathrm{cl}}^{\mathcal{O}}+\frac{1}{2 c}\left\langle\mathbf{j}(0)\left[\left(\mathcal{O}-\mathcal{O}^{\prime}\right) \times \mathbf{j}(t)\right]\right\rangle=C_{\mathrm{cl}}^{\mathcal{O}} \equiv C_{\mathrm{cl}}
$$

Even though all physical systems are finite, we have argued that simulating solid-state VCD requires imposing periodic boundary conditions $(\mathrm{PBC})$ on the structure model. Therein, the absolute position is ill-defined and the magnetic dipole moment is, therefore, evaluated in locally finite virtual cells, which corresponds to a distributed origin (DO) gauge. ${ }^{2,53,60}$ Yet to eventually ensure gauge invariance (Equation 5), it has to be translated to a common origin (CO). ${ }^{53,61}$ With this the total magnetic dipole moment is found as the sum of the individual 
contributions of virtual cells:

$$
\mathbf{m}^{\mathcal{O}}(t)=\sum_{i}\left(\mathbf{m}_{i}(t)+\frac{1}{2 c}\left(\mathbf{r}_{i}(t)-\mathcal{O}\right) \times \mathbf{j}_{i}(t)\right)
$$

where the set of $\mathbf{r}_{i}$ spans the distributed origin gauge for $\mathbf{m}_{i}$. Equation 6, however, is ill-defined for an infinite crystal.

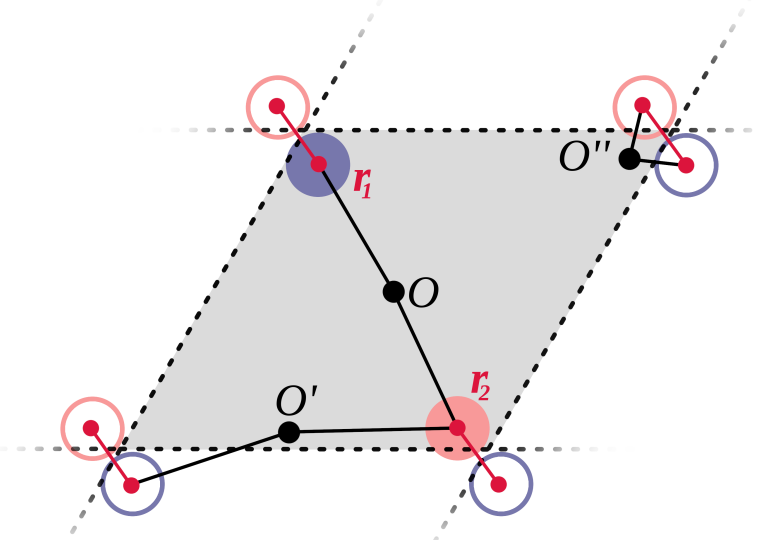

Figure 1: Sketch illustrating the common origin problem within periodic boundaries. Full circles are molecular units in the cell (rose and blue), while empty circles correspond to different periodic images of the same particles. The black dots represent three different choices of a common origin within the cell with black lines indicating the magnetic gauge, that is, the chosen pair of particles according to the nearest-image convention. Evidently, each origin takes different pairs of particles, which changes the computational result and introduces an additional origin-dependence into VCD. The new formalism presented here evaluates the nearest periodic image always directly between particles (red lines), which bypasses the origin.

Figure 1 illustrates the common origin problem that arises under PBC. The minimum image convention ensures that pair distances are always between adjacent (nearest) neighbours taking the periodic image if necessary. This convention can be expressed through a conditional lattice translation $\Delta_{\mathcal{O} i}^{\mathrm{PBC}}$ and the gauge vector in Equation 6 becomes:

$$
\mathbf{r}_{i}(t)-\mathcal{O} \stackrel{\mathrm{PBC}}{\longrightarrow} \mathbf{r}_{i}(t)-\mathcal{O}+\Delta_{\mathcal{O} i}^{\mathrm{PBC}}
$$

$\Delta_{\mathcal{O} i}^{\mathrm{PBC}}$ is a multiple of the lattice vectors translating $\mathbf{r}_{i}$ in order to yield the nearest image to 
the origin $\mathcal{O}$. Figure 1 exemplifies for two distributed units and three commons origins that depending on the choice of origin, different images of the units are considered (black lines). However, this additional origin dependence of $\mathbf{m}(t)$ does not vanish upon evaluation of the rotational strength since the value of $\Delta_{\mathcal{O} i}^{\mathrm{PBC}}$ is not a constant, but depends on the chosen unit $i$. Hence by casting the system into PBC, an artificial gauge dependence is introduced:

$$
C_{\mathrm{cl}}^{\mathcal{O}}=\langle\mathbf{j}(0) \mathbf{m}(t)\rangle+\frac{1}{2 c}\left\langle\mathbf{j}(0) \sum_{i}\left[\left(\mathbf{r}_{i}(t)-\mathcal{O}+\Delta_{\mathcal{O} i}^{\mathrm{PBC}}\right) \times \mathbf{j}_{i}(t)\right]\right\rangle \neq C_{\mathrm{cl}}^{\mathcal{O}^{\prime}}
$$

with $\mathbf{j}(t)=\sum_{i} \mathbf{j}_{i}(t)$ and $\mathbf{m}(t)=\sum_{i} \mathbf{m}_{i}(t)$. It is important to note that the artificial term would disappear if a complete, finite microcrystal were simulated. We have shown in the past that with this formalism fully periodic structure models can still be studied via averaging multiple origins, but the VCD spectrum is not truly gauge invariant. ${ }^{31,47}$ Furthermore, spectral signatures may have been missed or artificially introduced (further discussion as well as VCD spectra computed with different origins can be found in the SI).

We present a new formulation of the TCF that reconnects the infinite PBC model to the finite physical world. Taking advantage of the symmetric properties of $C_{\mathrm{cl}}$ it is possible to obtain an explicitly gauge-independent expression where the origin $\mathcal{O}$ is bypassed and only the vector $\left(\mathbf{r}_{j}(t)-\mathbf{r}_{i}(0)\right)$ is involved. In the result, the nearest image convention is taken directly between units (red lines in Figure 1), which leaves with the sole requirement, in analogy to the finite case, the origin be common to the distributed origins $\left(\mathbf{r}_{i}\right)$. Starting from Equation 8, the final expression for the TCF reads (see the SI for a detailed derivation):

$$
C_{\mathrm{cl}}=\langle\mathbf{j}(0) \mathbf{m}(t)\rangle+\frac{1}{2 c} \sum_{i}\left\langle\mathbf{j}_{i}(0) \sum_{j}\left[\left(\mathbf{r}_{j}(t)+\frac{1}{2} \Delta_{i j}^{\mathrm{PBC}}(0)\right) \times \mathbf{j}_{j}(t)\right]\right\rangle .
$$

where $\Delta_{i j}^{\mathrm{PBC}}$ indicates the nearest image convention between units $i$ and $j$ (see above).

is the vector, multiple of the unit cell vectors, that translates rj(0) to the minimum image of $\mathrm{j}$ with respect to ri(0), as in miminum image convention for interactions. The first term in represents the summed correlation of moments within and between spatial units in the 
distributed origin gauge; it thus contains local and non-local contributions to VCD alike. The second, gauge transport, term is the transformation from distributed to common origin, adapted for periodic boundary conditions, which is purely non-local. Unquestionably, both terms have to be considered to deliver the physically relevant result. With Equation 9 gauge invariance of the rotational strength is recovered within an infinite lattice, while non-local correlations can still be computed in the limits of the used simulation cell.

a)

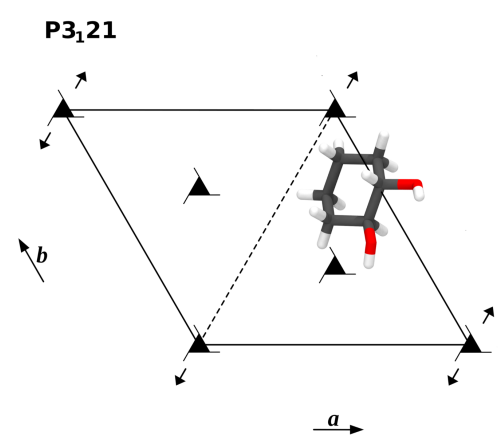

b)

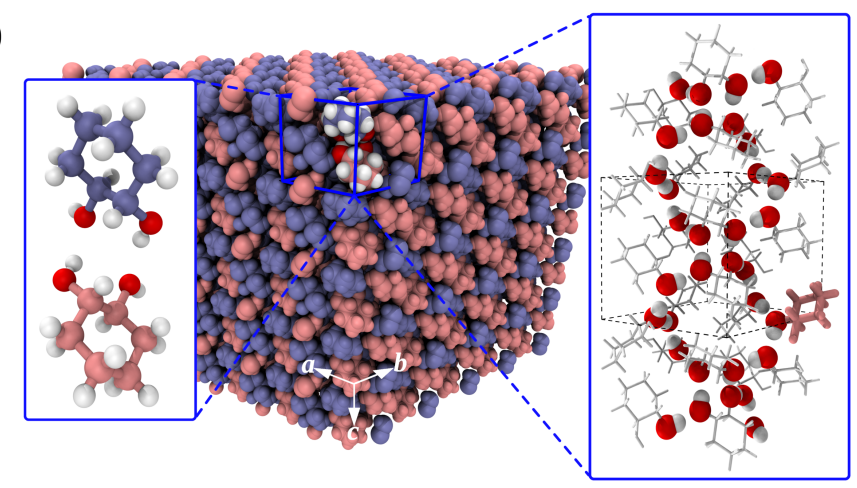

Figure 2: Left: Unit cell of $(1 S, 2 S)$-CHDO with asymmetric unit and main symmetry operations. ${ }^{62}$ The cyclic diol crystallises with trigonal lattice in the chiral space group $P 3_{1} 21$ containing a principal threefold screw axis and a global $C 2$ symmetry. Right: Starting from a $C 2$-symmetric dimer, a twisted three-dimensional $\mathrm{OH}$-network is formed that traverses the entire unit cell by means of $3_{1}$ rotary translation. For the AIMD simulations a single unit cell has been used (blue cage).

We added the new gauge algorithm to the ChirPy code, available on GitLab, ${ }^{63}$ and applied it to crystalline $(1 S, 2 S)$-CHDO. The cyclic diol crystallises with trigonal lattice in the chiral space group $P 3_{1} 21$ containing a principal threefold screw axis and a global $C 2$ symmetry (Figure 2, left). ${ }^{64}$ The asymmetric unit consists of a single $(1 S, 2 S)$-CHDO molecule that is propagated to form a dimer. The two hydroxyl groups are not equivalent, with one forming a stable intramolecular hydrogen bond and the other bridging to the neighbouring diol. While in gas phase the isolated monomer exists in two almost iso-energetic equatorial conformers, with either quasi-perpendicular or quasi-colinear $\mathrm{OOH}$ orientation, ${ }^{65}$ the latter is favoured in the crystal. In this way a twisted three-dimensional OH-network that traverses the entire unit cell can be formed by means of $3_{1}$ rotary translation (Figure 2 , 
right). Following the protocol in Scheme 1, we considered a single unit cell only, containing 120 atoms, and carried out FPMD calculations using the CP2K package ${ }^{66-68}$ with the hybridfunctional B3LYP ${ }^{69}$ and obtained the quantum response with the CPMD package by means of projected NVPT. ${ }^{47,70}$ Assembling the gauge under PBC, we choose whole molecules as the smallest unit of the distributed gauge that enters Equation 9, and compare the obtained spectra with solid-state VCD measurements of $(1 S, 2 S)$-CHDO in $\mathrm{KBr}$ (see the SI for the experimental methods and computational details).

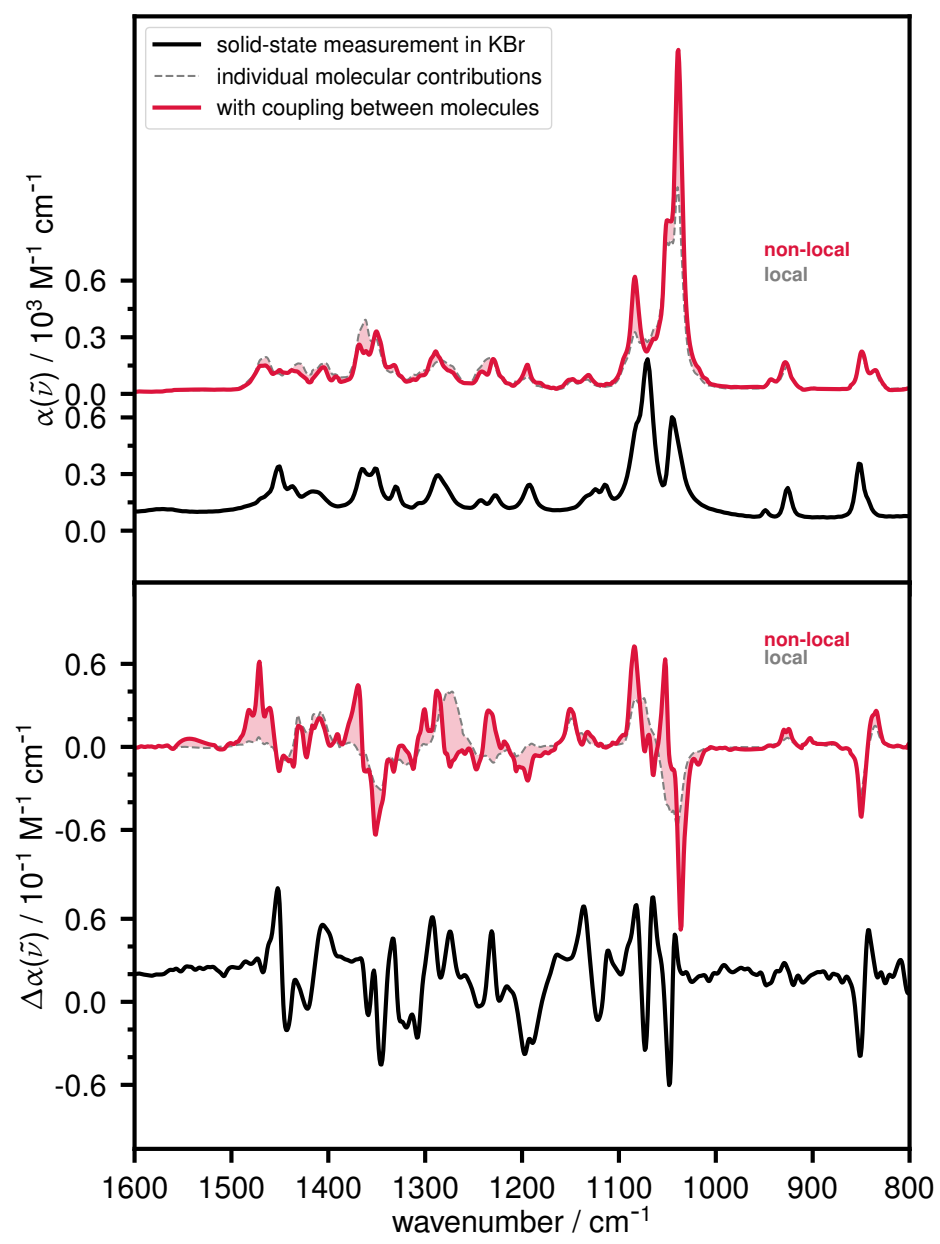

Figure 3: IR (top) and VCD (bottom) spectra of (1S,2S)-CHDO obtained with the gaugeinvariant formalism using FPMD simulations. The VCD spectrum is very rich in detail and strongly determined by non-local contributions from the chiral crystal. Peak alignment was done based on the IRSA algorithm originally developed by Riniker and co-workers. ${ }^{71}$

Figure 3 shows the computational and experimental results, for which we adapted the 
recently presented IRSA algorithm by Riniker and co-workers to continuous spectral densities. ${ }^{71}$ Hence, the computational peaks are aligned to their experimental pendants, using a scoring function for peak position and intensity, in order to facilitate the comparison between simulated and experimental spectra (see the SI for details). It is important to note that we choose to align the IR spectra, which is more reliable than seeking for an alignment of the congested VCD spectra, but nevertheless improves the significance of predicted VCD peaks.

The IR absorption spectrum is gauge-independent, but may include local and non-local contributions, distinguishing between correlations stemming only from the same molecule (or any other chosen smallest unit) as opposed to the full spectrum, including coupling between units. ${ }^{31,47}$ In the case of $(1 S, 2 S)$-CHDO, Figure 3 (top) reveals that indeed there are changes in IR intensity of certain peaks, but no overall change of the spectrum is observed. The overall strongest absorption in spectrum is found in the CO-stretching region $\left(1000-1100 \mathrm{~cm}^{-1}\right)$, where intermolecular coupling further increases the peak intensity. In the experiment this band appears as doublet from two non-equivalent hydroxyl groups with additional shoulders at high and low frequency from modes corresponding to combined CC-stretch and $\mathrm{CH}_{2^{-}}$ twist, which is in principal reproduced by the FPMD, but the predicted intensities are overestimated especially for the peak at lower frequency. For the remaining IR absorption spectrum the agreement with FPMD is almost impeccable, with only the mixing between CC-stretching and $\mathrm{CH}_{2}$-rocking $\left(850 \mathrm{~cm}^{-1}\right)$ showcasing two separated peaks in the simulation instead of a peak with shoulder in the experiment. The applied frequency scaling due to IRSA (cf. Figures S5 and S6) varies between spectral regions with hardly any shift resulting for the CO-stretching band, and a proposed red-shift for the remaining parts. Hence for actual peak assignment, post-processing the computational results with IRSA is more adapted to the individual deviation of frequencies from computed spectra than a single scaling factor for the entire spectrum.

The VCD spectrum of $(1 S, 2 S)$-CHDO is very rich in detail, which points to the conformational rigidity that is typical of molecular crystals. Furthermore, it is strongly determined 
by non-local contributions - much more than the IR absorption spectrum - as revealed by the computations (Figure 3, bottom), which is an indication of the crystal structure's strong influence (cf. Figure 2); in turn, the local scope, carried within individual molecules, cannot reproduce large parts of the spectrum. It is then not surprising that almost all bending vibrations involving the outer atoms of $(1 S, 2 S)$-CHDO, like the $\mathrm{CH}_{2}$-deformation (1450$1500 \mathrm{~cm}^{-1}$ ) and $\mathrm{OH}-/ \mathrm{CH}$-rocking $\left(1200-1400 \mathrm{~cm}^{-1}\right)$ bands, but also the CO-stretching bands, are tracers of the crystal's chirality. Especially, the CO-stretching band exhibits strong nonlocal effects with peaks appearing superposed, partially cancelling each other, which renders the VCD band very sensitive to small shifts in frequencies. Like it is the case for IR absorption, this VCD band is overestimated by the simulation; it is for these two reasons that this band is less reliable for assignment. In contrast, the CC-stretching band $\left(850 \mathrm{~cm}^{-1}\right)$ is a local feature and does not depend much on chiral crystal packing. Interestingly, it nevertheless exhibits an origin-dependence (cf. Figure S1) giving a good example of how significantly Equation 9 mediates the gauge between molecules under PBC. The $\mathrm{OH}-$ bending bands at approximately $1350 \mathrm{~cm}^{-1}$ appear as a couplet that is only partially encountered in the experimental spectrum, but the overall agreement of theory and experiment is very convincing in light of the small simulation cell that has been used. Figure 3 allows for an unambiguous assignment of the absolute configuration of $(1 S, 2 S)$-CHDO in the sample, but also for the verification of the investigated polymorph. ${ }^{64,72,73}$

Our results verify the new formalism allows for the computation of solid-state VCD in the periodic gauge. While the conventional equations of VCD in isolated systems are inapt for periodic boundaries as they depend on the chosen common origin, the new equations yield the desired gauge-invariant result. We are able to reproduce the very rich solid-state VCD spectrum of of $(1 S, 2 S)$-CHDO crystals already on the basis of a single FPMD run of one unit cell only. This is even more astounding as non-local contributions play an important role in this system reflecting the complex spatial organisation of molecules in the unit cell. Solid-state VCD can extend its explanatory power towards the determination of a molecule's 
crystal structure as soon as the computations no longer depend on crystallographic data as input. However, the capability to distinguish crystal modifications through VCD is already of pharmaceutical interest and merits due attention. ${ }^{1}$ We present herewith a straightforward workflow for solid-state VCD that can be integrated into industrial drug design schemes; a python code example and the required files to generate the spectra in this work, as well as a snapshot version of the ChirPy package are available online. ${ }^{63}$ Although FPMD simulations are too costly to be implemented into high-throughput schemes, it nevertheless delivers an almost black-box access to VCD of very realistic model systems and bulk phases. Nowadays with more and more computational resources available, workable FPMD implementations are omnipresent and their acceleration, for instance via machine learning or linear scaling schemes, target of ongoing research. ${ }^{68,74}$ An attractive alternative are polarisable force fields that can dramatically reduce the computational cost of MD and electromagnetic response (steps 1 and 2 in Scheme 1), ${ }^{75}$ and subsequently use the gauge formalism presented here. They have the potential to improve the statistical sampling with longer time scales and larger system size, but may suffer from a lack of accuracy. It should be noted that the presented gauge formalism can compute VCD in gas or liquid phase as well, but simplifications can be made therefor without loosing accuracy, leading to known theoretical formulations of VCD in finite boundaries (see SI). ${ }^{47}$ For large simulation cells, the pseudo-isolated approximation can

be imposed to clip chiral centres and to accelerate the evaluation of Equation 9. Although the presented theoretical treatise is done in the Heisenberg picture, it can be carried out in the Schrödinger picture as well, which would yield the corresponding terms for static calculations.

\section{Acknowledgement}

This work was granted access to the HPC resources of CINES under the allocation 2020-A9 made by GENCI This work has been supported by the French National Research Agency 
(ANR) Dichroprobe project (Grant ANR-18-CE29-0001-01).

\section{Supporting Information Available}

The following files are available free of charge.

- SI_manuscript.pdf: Theory (Rotational Strength for Periodic Boundaries), Experimental Methods, Computational Details

- md-nvt-1.restart: CP2K input file for AIMD calculations (step 1 in Scheme 1

- cpmd_job.inp: CPMD input file for NVPT calculations (step 2 in Scheme 1

\section{Data Available}

We have developed the python package ChirPy that is available online (http://doi.org/ 10.5281/zenodo.4775330), and provide all data needed to reproduce the spectra shown in this manuscript (without IRSA) via https://doi.org/10.5281/zenodo. 4776907.

\section{References}

(1) Nafie, L. A. Vibrational Optical Activity: From Discovery and Development to Future Challenges. Chirality 2020, 32, 667-692.

(2) Nafie, L. A. Vibrational Optical Activity: Principles and Applications; John Wiley \& Sons Ltd., 2011.

(3) Holzwarth, G.; Hsu, E. C.; Mosher, H. S.; Faulkner, T. R.; Moscowitz, A. Infrared Circular Dichroism of Carbon-Hydrogen and Carbon-Deuterium Stretching Modes. Observations. J. Am. Chem. Soc. 1974, 96, 251-252. 
(4) Krupová, M.; Kessler, J.; Bouř, P. Recent Trends in Chiroptical Spectroscopy: Theory and Applications of Vibrational Circular Dichroism and Raman Optical Activity. ChemPlusChem 2020, 85, 561-575.

(5) Freedman, T. B.; Cao, X.; Dukor, R. K.; Nafie, L. A. Absolute Configuration Determination of Chiral Molecules in the Solution State Using Vibrational Circular Dichroism. Chirality 2003, 15, 743-758.

(6) Wesolowski, S. S.; Pivonka, D. E. A Rapid Alternative to X-Ray Crystallography for Chiral Determination: Case Studies of Vibrational Circular Dichroism (VCD) to Advance Drug Discovery Projects. Bioorg. Med. Chem. Lett. 2013, 23, 4019-4025.

(7) Polavarapu, P. L.; Santoro, E. Vibrational Optical Activity for Structural Characterization of Natural Products. Nat. Prod. Rep. 2020, 37, 1661-1699.

(8) Burgueño-Tapia, E.; Joseph-Nathan, P. Vibrational Circular Dichroism: Recent Advances for the Assignment of the Absolute Configuration of Natural Products. Nat. Prod. Commun. 2017, 12, 641-651.

(9) Bogaerts, J.; Desmet, F.; Aerts, R.; Bultinck, P.; Herrebout, W.; Johannessen, C. A Combined Raman Optical Activity and Vibrational Circular Dichroism Study on Artemisinin-Type Products. Phys. Chem. Chem. Phys. 2020, 22, 18014-18024.

(10) Ouyang, J.; Swartjes, A.; Geerts, M.; Gilissen, P. J.; Wang, D.; Teeuwen, P. C. P.; Tinnemans, P.; Vanthuyne, N.; Chentouf, S.; Rutjes, F. P. J. T. et al. Absolute Configuration and Host-Guest Binding of Chiral Porphyrin-Cages by a Combined Chiroptical and Theoretical Approach. Nat. Commun. 2020, 11, 4776.

(11) Sato, H.; Kawamura, I.; Yamagishi, A.; Sato, F. Solid-State Vibrational Circular Dichroism Spectra of Isoleucine and Its Related Compounds: Effects of Interplay between Two Chiral Centers. Chem. Lett. 2017, 46, 449-452. 
(12) Bouchet, A.; Brotin, T.; Cavagnat, D.; Buffeteau, T. Induced Chiroptical Changes of a Water-Soluble Cryptophane by Encapsulation of Guest Molecules and Counterion Effects. Chem. Eur. J. 2010, 16, 4507-4518.

(13) Choi, H.; Cho, K. J.; Seo, H.; Ahn, J.; Liu, J.; Lee, S. S.; Kim, H.; Feng, C.; Jung, J. H. Transfer and Dynamic Inversion of Coassembled Supramolecular Chirality through 2DSheet to Rolled-up Tubular Structure. J. Am. Chem. Soc. 2017, 139, 17711-17714.

(14) Gautier, C.; Bürgi, T. Chiral Gold Nanoparticles. ChemPhysChem 2009, 10, 483-492.

(15) Roy Bhattacharya, S.; Bürgi, T. Amplified Vibrational Circular Dichroism as a Manifestation of the Interaction between a Water Soluble Gold Nanocluster and Cobalt Salt. Nanoscale 2019, 11, 23226-23233.

(16) Merten, C. Recent Advances in the Application of Vibrational Circular Dichroism Spectroscopy for the Characterization of Asymmetric Catalysts. Eur. J. Org. Chem. 2020, 2020, 5892-5900.

(17) Golub, T. P.; Merten, C. Stereochemistry of the Reaction Intermediates of Prolinol Ether Catalyzed Reactions Characterized by Vibrational Circular Dichroism Spectroscopy. Chem. - Eur. J. 2020, 26, 2349-2353.

(18) Oulevey, P.; Luber, S.; Varnholt, B.; Bürgi, T. Symmetry Breaking in Chiral Ionic Liquids Evidenced by Vibrational Optical Activity. Angewandte Chemie International Edition 2016, 55, 11787-11790.

(19) Koenis, M. A. J.; Nicu, V. P.; Visscher, L.; Kuehn, C.; Bremer, M.; Krier, M.; Untenecker, H.; Zhumaev, U.; Küstner, B.; Buma, W. J. Vibrational Circular Dichroism Studies of Exceptionally Strong Chirality Inducers in Liquid Crystals. Phys. Chem. Chem. Phys. 2021, 23, 10021-10028. 
(20) Ma, S.; Cao, X.; Mak, M.; Sadik, A.; Walkner, C.; Freedman, T. B.; Lednev, I. K.; Dukor, R. K.; Nafie, L. A. Vibrational Circular Dichroism Shows Unusual Sensitivity to Protein Fibril Formation and Development in Solution. J. Am. Chem. Soc. 2007, 129, 12364-12365.

(21) Keiderling, T. A. Structure of Condensed Phase Peptides: Insights from Vibrational Circular Dichroism and Raman Optical Activity Techniques. Chem. Rev. 2020, 120, $3381-3419$.

(22) Measey, T. J.; Schweitzer-Stenner, R. Vibrational Circular Dichroism as a Probe of Fibrillogenesis: The Origin of the Anomalous Intensity Enhancement of Amyloid-like Fibrils. J. Am. Chem. Soc. 2011, 133, 1066-1076.

(23) Koenis, M. A. J.; Xia, Y.; Domingos, S. R.; Visscher, L.; Buma, W. J.; Nicu, V. P. Taming Conformational Heterogeneity in and with Vibrational Circular Dichroism Spectroscopy. Chem. Sci. 2019, 10, 7680-7689.

(24) Le Barbu-Debus, K.; Bowles, J.; Jähnigen, S.; Clavaguéra, C.; Calvo, F.; Vuilleumier, R.; Zehnacker, A. Assessing Cluster Models of Solvation for the Description of Vibrational Circular Dichroism Spectra: Synergy between Static and Dynamic Approaches. Phys. Chem. Chem. Phys. 2020, 22, 26047-26068.

(25) Nicu, V. P.; Baerends, E. J. On the Origin Dependence of the Angle Made by the Electric and Magnetic Vibrational Transition Dipole Moment Vectors. Phys. Chem. Chem. Phys. 2011, 13, 16126-16129.

(26) Perera, A. S.; Cheramy, J.; Poopari, M. R.; Xu, Y. Aggregation of Lactic Acid in Cold Rare-Gas Matrices and the Link to Solution: A Matrix Isolation-Vibrational Circular Dichroism Study. Phys. Chem. Chem. Phys. 2019, 21, 3574-3584.

(27) Légrády, B.; Vass, E.; Tarczay, G. Matrix-Isolation Vibrational Circular Dichroism 
Spectroscopy in Structural Studies of Peptides: Conformational Landscape of the Ac(Ala)1-4-OMe Depsipeptide Series. Journal of Molecular Spectroscopy 2018, 351, 29-38.

(28) Pollok, C. H.; Merten, C. Conformational Distortion of $\alpha$-Phenylethyl Amine in Cryogenic Matrices - a Matrix Isolation VCD Study. Phys. Chem. Chem. Phys. 2016, 18, 13496-13502.

(29) Pollok, C. H.; Riesebeck, T.; Merten, C. Photoisomerization of a Chiral Imine Molecular Switch Followed by Matrix-Isolation VCD Spectroscopy. Angew. Chem. Int. Ed. 2017, $56,1925-1928$.

(30) Merten, C.; Xu, Y. Chirality Transfer in a Methyl Lactate-Ammonia Complex Observed by Matrix-Isolation Vibrational Circular Dichroism Spectroscopy. Angew. Chem. Int. Ed. 2013, 52, 2073-2076.

(31) Jähnigen, S.; Scherrer, A.; Vuilleumier, R.; Sebastiani, D. Chiral Crystal Packing Induces Enhancement of Vibrational Circular Dichroism. Angew. Chem. Int. Ed. 2018, 57, 13344-13348.

(32) Pérez-Mellor, A.; Zehnacker, A. Vibrational Circular Dichroism of a 2,5Diketopiperazine (DKP) Peptide: Evidence for Dimer Formation in Cyclo LL or LD Diphenylalanine in the Solid State. Chirality 2017, 29, 89-96.

(33) Pérez-Mellor, A.; Barbu-Debus, K. L.; Zehnacker, A. Solid-State Synthesis of Cyclo LD-Diphenylalanine: A Chiral Phase Built from Achiral Subunits. Chirality 2020, 32, 693-703.

(34) Sato, H.; Kawamura, I. Solid-State Vibrational Circular Dichroism Studies on the Conformation of an Amino Acid Molecule in Crystalline State. Biochimica et Biophysica Acta (BBA) - Proteins and Proteomics 2020, 1868, 140439. 
(35) Quesada-Moreno, M. M.; Aviles-Moreno, J. R.; Lopez-Gonzalez, J. J.; Jacob, K.; Vendier, L.; Etienne, M.; Alkorta, I.; Elguero, J.; Claramunt, R. M. Supramolecular Organization of Perfluorinated 1H-Indazoles in the Solid State Using X-Ray Crystallography, SSNMR and Sensitive (VCD) and Non Sensitive (MIR, FIR and Raman) to Chirality Vibrational Spectroscopies. Phys. Chem. Chem. Phys. 2017, 19, 1632-1643.

(36) Barron, L. D. Molecular Light Scattering and Optical Activity; Cambridge University Press: Cambridge, 2004.

(37) Merten, C.; Kowalik, T.; Hartwig, A. Vibrational Circular Dichroism Spectroscopy of Solid Polymer Films: Effects of Sample Orientation. Appl Spectrosc 2008, 62, 901-905.

(38) Buffeteau, T.; Lagugné-Labarthet, F.; Sourisseau, C. Vibrational Circular Dichroism in General Anisotropic Thin Solid Films: Measurement and Theoretical Approach. Appl. Spectrosc., AS 2005, 59, 732-745.

(39) Frelek, J.; Górecki, M.; Łaszcz, M.; Suszczyńska, A.; Vass, E.; Szczepek, W. J. Distinguishing between Polymorphic Forms of Linezolid by Solid-Phase Electronic and Vibrational Circular Dichroism. Chem. Commun. 2012, 48, 5295-5297.

(40) Pazderková, M.; Pazderka, T.; Shanmugasundaram, M.; Dukor, R. K.; Lednev, I. K.; Nafie, L. A. Origin of Enhanced VCD in Amyloid Fibril Spectra: Effect of Deuteriation and pH. Chirality 2017, 29, 469-475.

(41) Jiang, N.; Tan, R. X.; Ma, J. Simulations of Solid-State Vibrational Circular Dichroism Spectroscopy of (S)-Alternarlactam by Using Fragmentation Quantum Chemical Calculations. J. Phys. Chem. B 2011, 115, 2801-2813.

(42) Gonzalez, J. J. L.; Urena, F. P.; Moreno, J. R. A.; Mata, I.; Molins, E.; Claramunt, R. M.; Lopez, C.; Alkorta, I.; Elguero, J. The Chiral Structure of 1H-Indazoles in the Solid State: A Crystallographic, Vibrational Circular Dichroism and Computational Study. New J. Chem. 2012, 36, 749-758. 
(43) Declerck, V.; Pérez-Mellor, A.; Guillot, R.; Aitken, D. J.; Mons, M.; Zehnacker, A. Vibrational Circular Dichroism as a Probe of Solid-state Organisation of Derivatives of Cyclic B-amino Acids: Cis - and Trans -2-aminocyclobutane-1-carboxylic Acid. Chirality 2019, 31, 547-560.

(44) Kubo, R. Statistical-Mechanical Theory of Irreversible Processes. i. General Theory and Simple Applications to Magnetic and Conduction Problems. J. Phys. Soc. Jpn. 1957, 12, 570-586.

(45) Ramírez, R.; López-Ciudad, T.; Kumar P, P.; Marx, D. Quantum Corrections to Classical Time-Correlation Functions: Hydrogen Bonding and Anharmonic Floppy Modes. The Journal of Chemical Physics 2004, 121, 3973-3983.

(46) Abbate, S.; Longhi, G.; Kwon, K.; Moscowitz, A. The Use of Cross-Correlation Functions in the Analysis of Circular Dichroism Spectra. J. Chem. Phys. 1998, 108, 50-62.

(47) Scherrer, A.; Vuilleumier, R.; Sebastiani, D. Vibrational Circular Dichroism from Ab Initio Molecular Dynamics and Nuclear Velocity Perturbation Theory in the Liquid Phase. J. Chem. Phys. 2016, 145, 084101.

(48) Born, M.; Oppenheimer, R. Zur Quantentheorie Der Molekeln. Ann. Phys. 1927, 389, $457-484$.

(49) Cheeseman, J.; Frisch, M.; Devlin, F.; Stephens, P. Ab Initio Calculation of Atomic Axial Tensors and Vibrational Rotational Strengths Using Density Functional Theory. Chem. Phys. Lett. 1996, 252, 211-220.

(50) Reiter, K.; Kühn, M.; Weigend, F. Vibrational Circular Dichroism Spectra for Large Molecules and Molecules with Heavy Elements. The Journal of Chemical Physics 2017, 146,054102 . 
(51) Nicu, V. P.; Neugebauer, J.; Wolff, S. K.; Baerends, E. J. A Vibrational Circular Dichroism Implementation within a Slater-Type-Orbital Based Density Functional Framework and Its Application to Hexa- and Hepta-Helicenes. Theor Chem Account 2008, 119, $245-263$.

(52) Nafie, L. A.; Freedman, T. B. Vibronic Coupling Theory of Infrared Vibrational Transitions. J. Chem. Phys. 1983, 78, 7108-7116.

(53) Scherrer, A.; Vuilleumier, R.; Sebastiani, D. Nuclear Velocity Perturbation Theory of Vibrational Circular Dichroism. J. Chem. Theory Comput. 2013, 9, 5305-5312.

(54) Scherrer, A.; Agostini, F.; Sebastiani, D.; Gross, E. K. U.; Vuilleumier, R. Nuclear Velocity Perturbation Theory for Vibrational Circular Dichroism: An Approach Based on the Exact Factorization of the Electron-Nuclear Wave Function. J. Chem. Phys. 2015, 143, 074106.

(55) Thomas, M.; Kirchner, B. Classical Magnetic Dipole Moments for the Simulation of Vibrational Circular Dichroism by Ab Initio Molecular Dynamics. J. Phys. Chem. Lett. 2016, 7, 509-513.

(56) Rosenfeld, L. Quantenmechanische Theorie der natürlichen optischen Aktivität von Flüssigkeiten und Gasen. Z. Für Phys. 1929, 52, 161-174.

(57) Hirschfelder, J. O. Classical and Quantum Mechanical Hypervirial Theorems. J. Chem. Phys. 1960, 33, 1462-1466.

(58) Gaigeot, M.-P.; Martinez, M.; Vuilleumier, R. Infrared Spectroscopy in the Gas and Liquid Phase from First Principle Molecular Dynamics Simulations: Application to Small Peptides. Molecular Physics 2007, 105, 2857-2878.

(59) Kubo, R.; Toda, M.; Hashitsume, N. In Statistical Physics II; Cardona, M., Fulde, P., 
Queisser, H.-J., Eds.; Springer Series in Solid-State Sciences; Springer Berlin Heidelberg: Berlin, Heidelberg, 1985; Vol. 31.

(60) Sebastiani, D.; Parrinello, M. A New Ab-Initio Approach for NMR Chemical Shifts in Periodic Systems. J. Phys. Chem. A 2001, 105, 1951-1958.

(61) Nicu, V. P. Revisiting an Old Concept: The Coupled Oscillator Model for VCD. Part 1: The Generalised Coupled Oscillator Mechanism and Its Intrinsic Connection to the Strength of VCD Signals. Phys. Chem. Chem. Phys. 2016, 18, 21202-21212.

(62) Birkbeck College, University of London, Crystallographic Space Group Diagrams and Tables. 1997; http://img.chem.ucl.ac.uk/sgp/mainmenu.htm.

(63) Jähnigen, S. ChirPy - A Python Package for Analysing Supramolecular and Electronic Structure, Chirality and Dynamics (Version 0.21.5). Zenodo, 2021; http://doi .org/ $10.5281 /$ zenodo .4775330 .

(64) Bebiano, S. V. S.; Rosado, M. T. S.; Castro, R. A. E.; Ramos Silva, M.; Canotilho, J.; Maria, T. M. R.; Eusébio, M. E. S. Polymorphism of Cis-1,4-Cyclohexanediol, a New Plastic Crystal Former. Considerations on Isomeric Cyclohexanediols Plastic Crystal Forming Abilities. Journal of Molecular Structure 2014, 1078, 10-19.

(65) Hartwig, B.; Lange, M.; Poblotzki, A.; Medel, R.; Zehnacker, A.; Suhm, M. A. The Reduced Cohesion of Homoconfigurational 1,2-Diols. Phys. Chem. Chem. Phys. 2020, 22, 1122-1136.

(66) VandeVondele, J.; Krack, M.; Mohamed, F.; Parrinello, M.; Chassaing, T.; Hutter, J. QUICKSTEP: Fast and Accurate Density Functional Calculations Using a Mixed Gaussian and Plane Waves Approach. Comput. Phys. Commun. 2005, 167, 103-128.

(67) The CP2K Developers Group, 2001-2021. https://www.cp2k.org. 
(68) Kühne, T. D.; Iannuzzi, M.; Del Ben, M.; Rybkin, V. V.; Seewald, P.; Stein, F.; Laino, T.; Khaliullin, R. Z.; Schütt, O.; Schiffmann, F. et al. CP2K: An Electronic Structure and Molecular Dynamics Software Package - Quickstep: Efficient and Accurate Electronic Structure Calculations. J. Chem. Phys. 2020, 152, 194103.

(69) Stephens, P. J.; Devlin, F. J.; Chabalowski, C. F.; Frisch, M. J. Ab Initio Calculation of Vibrational Absorption and Circular Dichroism Spectra Using Density Functional Force Fields. J. Phys. Chem. 1994, 98, 11623-11627.

(70) CPMD 4.1.0, Copyright 2000-2019. IBM Corp. and by Max Planck Institute, Stuttgart, https://www. cpmd.org.

(71) Böselt, L.; Dötzer, R.; Steiner, S.; Stritzinger, M.; Salzmann, S.; Riniker, S. Determining the Regiochemistry and Relative Stereochemistry of Small and Druglike Molecules Using an Alignment Algorithm for Infrared Spectra. Anal. Chem. 2020, 92, 9124-9131.

(72) Leitão, M. L. P.; Castro, R. A. E.; Costa, F. S.; Redinha, J. S. Phase Transitions of 1,2-Cyclohexanediol Isomers Studied by Polarised Light Microscopy and Differential Thermal Analysis. Thermochimica Acta 2001, 378, 117-124.

(73) A Calorimetric Study of Phase Transitions for Some Cyclohexanediols. Thermochim. Acta 1995, 269-270, 405-413.

(74) Gastegger, M.; Behler, J.; Marquetand, P. Machine Learning Molecular Dynamics for the Simulation of Infrared Spectra. Chem. Sci. 2017, 8, 6924-6935.

(75) Semrouni, D.; Sharma, A.; Dognon, J.-P.; Ohanessian, G.; Clavaguéra, C. Finite Temperature Infrared Spectra from Polarizable Molecular Dynamics Simulations. J. Chem. Theory Comput. 2014, 10, 3190-3199. 